\title{
Geometrical phases for the $G(4,2)$ Grassmannian manifold
}

\author{
Regina Karle ${ }^{1}$ and Jiannis Pachos ${ }^{2,3}$ \\ 1 Ludwig-Maximilians-Universität, München, Germany \\ ${ }^{2}$ Max-Planck-Institut für Quantenoptik, D-85748 Garching, Germany \\ ${ }^{3}$ Optics Section, Blackett Laboratory, Imperial College London, London SW7 2BZ, U.K. *
}

(November 7, 2018)

\begin{abstract}
We generalize the usual abelian Berry phase generated for example in a system with two nondegenerate states to the case of a system with two doubly degenerate energy eigenspaces. The parametric manifold describing the space of states of the first case is formally given by the $G(2,1)$ Grassmannian manifold, while for the generalized system it is given by the $G(4,2)$ one. For the latter manifold which exhibits a much richer structure than its abelian counterpart we calculate the connection components, the field strength and the associated geometrical phases that evolve nontrivially both of the degenerate eigenspaces. A simple atomic model is proposed for their physical implementation.
\end{abstract}

PACS numbers: 03.65.Vf, 03.67.-a, 42.50.Vk

\section{INTRODUCTION}

Geometrical phases have attracted much interest since the seminal work by Berry [1]. The simple example of the abelian Berry phase produced, e.g. by the adiabatic transition of a spin- $1 / 2$ particle that follows a rotating magnetic field, has found many applications in quantum optics, molecular physics and so on. Theoretically, it was extended to non-abelian phases by Wilczek and Zee [2] by additionally employing to the setup of the usual Berry phase a degenerate structure that allows geometrical unitary evolutions of higher dimensionality describing transitions of population within the degenerate eigenspace. There are different applications of geometrical evolutions in the literature [3] and in particular related to quantum information. Special attention has been given to the evolutions that are parameterized by the $n$-dimensional complex projective manifold, $C P^{n}$. It corresponds to the parametric manifold $\mathcal{M}$ of a physical model where $n$ states out of $n+1$ are preserved degenerate at all times [4]. For this case the parametric space $\mathcal{M}$ is given by

$$
C P^{n} \approx \frac{U(n+1)}{U(n) \times U(1)},
$$

where the dictated control transformations are between the non-degenerate state and each degenerate one. By performing adiabatically closed paths in this parametric space geometrical unitary operators are generated, called holonomies, $\Gamma$, acting solely on the degenerate states. Their relevance for performing quantum computation was first demonstrated in [5]. Since then they enjoyed theoretical applications in quantum optical models with laser beams [6], trapped ions [7], optical cavities [8] or quantum dots [9].

A further generalization of the parametric control space is realized by employing the Grassmannian manifolds. They are given by the coset space structure

$$
G(m, n) \approx \frac{U(m)}{U(n) \times U(m-n)} .
$$

The complex projective manifolds can be considered as a special case of the Grassmannian ones. In particular, for $m=2$ and $n=1$ we obtain the $C P^{1}$ space where the Berry phases are defined, while for $m=n+1$ we have the identity $G(n+1, n) \approx C P^{n}$. There are two degenerate eigenspaces corresponding to the $G(m, n)$ model that are $n$ and $m-n$ dimensional. With the same adiabatic control procedure in the parametric space $\mathcal{M}=G(m, n)$ non-trivial holonomies are generated in both of them, contrary to all previously studied examples. Here, we shall restrict to the $G(4,2)$ case, where the connection components, the corresponding field strengths and a set of holonomies will be explicitly given.

*Present address. Email: jiannis.pachos@imperial.ac.uk 


\section{HOLONOMIES AND PHYSICAL MODELS}

\section{A. Berry phases}

The well known Berry phase can be produced by performing loops in the $G(2,1) \approx C P^{1}$ parametric space of external control parameters that determine the unitary evolution of two level system as sketched in Figure 1(a). Let us present one possible Berry phase implementation in atomic physics.

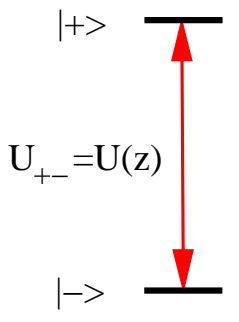

(a)

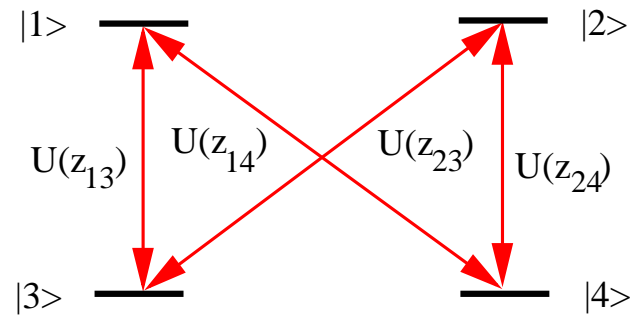

(b)

FIG. 1. Schematic state-structure and interactions parameterized by (a) the $G(2,1)$ and for (b) the $G(4,2)$ Grassmannian manifolds. The arrows represent $U(2)$ transformations between the connected states implemented e.g., by Raman transitions, while due to the coset structure there are no transformations between the degenerate states of (b).

Consider the case of an atom with two non-degenerate ground levels $|+\rangle$ and $|-\rangle$, with corresponding eigenvalues $E_{+}=\omega / 2$ and $E_{-}=-\omega / 2$, and an excited one $|e\rangle$. By performing a Raman adiabatic transfer [10] between levels $|+\rangle,|-\rangle$ with the help of two detuned laser fields with Rabi frequencies $\Omega_{+}$and $\Omega_{-}$and common detuning $\Delta$ it is possible to adiabatically eliminate level $|e\rangle$ and create in the basis $\{|+\rangle,|-\rangle\}$ the following unitary transformation

$$
\mathcal{U}(\theta, \phi)=\left(\begin{array}{cc}
\cos \frac{\theta}{2} & i e^{i \phi} \sin \frac{\theta}{2} \\
i e^{-i \phi} \sin \frac{\theta}{2} & \cos \frac{\theta}{2}
\end{array}\right)
$$

where $\theta=2\left|\Omega_{\text {eff }}\right| t, \phi=\arg \left\{\Omega_{\text {eff }}\right\}$ and $\Omega_{\text {eff }}=\Omega_{+} \Omega_{-}^{*} /(4 \Delta)$. In the above evolution we have neglected the A.C. Stark shift effect which can be compensated with properly detuned lasers. In accordance the initial free Hamiltonian of the $|+\rangle$ and $|-\rangle$ states, given by $H_{0}=\frac{\omega}{2} \sigma_{z}$, is transformed as

$$
H=\frac{\omega}{2} \mathcal{U}(\theta, \phi) \sigma_{z} \mathcal{U}^{\dagger}(\theta, \phi)
$$

Assume that at time $t=0$ we have $\theta=\phi=0$ and the initial state of the system is given by $\left|\Psi_{ \pm}(0)\right\rangle=| \pm\rangle$. Let us consider the evolution resulting when a closed path $C$ is spanned very slowly in the parametric plane $(\theta, \phi)$ in time interval $T$. Due to cyclicity and adiabaticity no population has moved from one state to the other at the end of the loop, that is, the energy of the system has not changed. Still the states are allowed to obtain a geometrical phase factor at the end of this evolution, which can be calculated from the time evolution operator of the system given by the Schrödinger equation. A dynamical phase does not appear due to the geodesic character of Raman evolutions. In particular, we obtain that $\left|\Psi_{+}(T)\right\rangle=\Gamma_{A}^{+}(C)|+\rangle$ and $\left|\Psi_{-}(T)\right\rangle=\Gamma_{A}^{-}(C)|-\rangle$ that is the states acquire a geometrical phase factor that depend on the spanned loop $C$, and a connection $A$. The components of the latter are defined, and analytically given for this case, by

$$
A_{\theta}^{ \pm}=\left\langle \pm\left|\mathcal{U}^{\dagger} \frac{\partial}{\partial \theta} \mathcal{U}\right| \pm\right\rangle=0 \quad \text { and } \quad A_{\phi}^{ \pm}=\left\langle \pm\left|\mathcal{U}^{\dagger} \frac{\partial}{\partial \phi} \mathcal{U}\right| \pm\right\rangle= \pm i \sin ^{2} \theta
$$

In terms of the connection $A$ the phase factors $\Gamma_{A}^{ \pm}(C) \equiv e^{i \varphi_{ \pm}}$are given by

$$
\Gamma_{A}^{ \pm}(C)=\exp \oint_{C} A^{ \pm}=\exp \iint_{\Sigma(C)} d \theta d \phi F_{\theta \phi}^{ \pm}(\theta, \phi)
$$


where $F_{\theta \phi}^{ \pm}(\theta, \phi)=\partial_{\theta} A_{\phi}^{ \pm}-\partial_{\phi} A_{\theta}^{ \pm}= \pm i \sin 2 \theta$ is the non-zero component of the field strength associated with $A$ and $\Sigma(C)$ is the surface on the plane $(\theta, \phi)$ bounded by the loop $C$. The second step in (1) is due to Stokes theorem. For any $C$ we obtain the relation $\varphi_{+}=-\varphi_{-}=\iint_{\Sigma(C)} d \theta d \phi \sin 2 \theta$ between the two Berry phases. Note that $\varphi_{+}=\Omega / 2$ where $\Omega$ is the solid angle spanned by the circulation of a unit vector with directions given by the $(\theta, \phi)$ angles. The half factor reflects the spin- $1 / 2$ transformation properties of the employed two atomic levels. This physical evolution produces a measurable abelian Berry phase. Their generalization to unitary matrices (holonomies) with the employment of degenerate structures is given in the following for the case of the Grassmannian manifold $G(4,2)$.

\section{B. Holonomies for the Grassmannian manifold $G(4,2)$}

The Grassmannian manifold $G(4,2)$ corresponds to the parametric space of the Hamiltonian $H=\mathcal{U} H_{0} \mathcal{U}^{\dagger}$ where $H_{0}=\omega / 2 \operatorname{diag}(1,1,-1,-1)$ and $\mathcal{U}$ are $U(4)$ unitary transformations that act non-trivially on the Hamiltonian $H_{0}$. Clearly, $H_{0}$ has the following eigenvectors $\left|{ }_{1}\right\rangle \equiv|1\rangle=(1,0,0,0),\left|+_{2}\right\rangle \equiv|2\rangle=(0,1,0,0),\left|-{ }_{1}\right\rangle \equiv|3\rangle=(0,0,1,0)$ and $\left|-_{2}\right\rangle \equiv|4\rangle=(0,0,0,1)$. The first two states span the degenerate eigenspace $S_{+}$with eigenvalue $E_{+}=\omega / 2$, while the last two span the eigenspace $S_{-}$with eigenvalue $E_{-}=-\omega / 2$. The schematic representation of this model is given in Figure 1(b), where the eligible transformations are depicted by arrows. Each arrow correspond to a $U(2)$ transformation $U\left(z_{i j}\right)$ parameterized by a complex number $z_{i j}=\theta_{i j} \exp i \phi_{i j}$ for $i=1,2$ and $j=3,4$. Hence, the real decomposition of the parametric space $G(4,2)$ is given by $\mathcal{M} \equiv\left\{\theta_{i j}, \phi_{i j}\right\} \equiv\left\{\sigma_{i j}\right\}$. Here we adopt the following ordering for the general unitary transformation, $\mathcal{U}(\sigma)=U\left(z_{13}\right) U\left(z_{14}\right) U\left(z_{23}\right) U\left(z_{24}\right)$.

Now we can define the connection components for each degenerate eigenspace. They are given from the following equation

$$
\left(A_{\sigma}^{ \pm}\right)_{\alpha \beta} \equiv\left\langle\alpha\left|\mathcal{U}^{\dagger}(\sigma) \frac{\partial}{\partial \sigma} \mathcal{U}(\sigma)\right| \beta\right\rangle
$$

where the basis vector $|\alpha\rangle$ and $|\beta\rangle$ belong in the same degenerate eigenspace of $H_{0}$. From (2) we see that the matrix $A_{\sigma}^{ \pm}$is anti-hermitian. For $\{\alpha, \beta\}=\{1,2\}$ we obtain the elements of the $2 \times 2$ matrix $A^{+}$, while for $\{\alpha, \beta\}=\{3,4\}$ we obtain the elements of $A^{-}$. The holonomic unitary operator, generated when a closed path, $C$, is spanned adiabatically in the space of the control parameters $\mathcal{M}$, is defined by

$$
\Gamma_{A}^{ \pm}(C) \equiv \mathbf{P} \exp \oint_{C} A^{ \pm}
$$

The path ordering symbol does not allow to calculate the path integral first and then to exponentiate the result as the different components of the connection $A$ do not commute in general. For those cases the simple form of Stokes theorem does not apply [11]. Still it is possible to analytically calculate the holonomies which result from the commuting components of the connection $A$ in the same way as we did for the abelian case. Alternatively, the non-abelian version of Stokes theorem can be employed as in $[6,12]$.

Before moving to the analytic calculation of the holonomies, let us see how the transformations $\mathcal{U}(\sigma)$ can be physically realized for example by an atomic system. According to Figure 1(b) we can generalize the model of the two level atom with four levels, pair-wise degenerate and apply laser beams connecting these states via additional exciting states by Raman adiabatic transfers. As in the case of the two-level system presented in the previous subsection, a $U(2)$ unitary transformation results from each Raman transition. Successive applications of those unitaries are able to construct the general unitary transformation $\mathcal{U}(\sigma)$ parameterized by the $G(4,2)$ manifold. A detailed study of the generation of the Berry phases with $\mathcal{M}=C P^{2}$ control manifold with an atomic system manipulated by Raman transitions is given in [13].

\section{CONNECTION AND FIELD STRENGTH COMPONENTS}

\section{A. Connection $A$}

In this subsection we shall present the connection components related to the parametric space $G(4,2)$ by employing definition (2). In particular, they are $2 \times 2$ matrices paired in the following with respect to the $S_{+}$and $S_{-}$degenerate eigenspaces they act on. Thus, we obtain 


$$
\begin{gathered}
A_{\theta_{13}}^{+}=\sin \theta_{23} \cos \theta_{14} \cos \theta_{24}\left(\begin{array}{cc}
0 & -e^{i\left(\phi_{13}-\phi_{23}\right)} \\
e^{-i\left(\phi_{13}-\phi_{23}\right)} & 2 i \sin \left(\phi_{13}-\phi_{14}+\phi_{24}-\phi_{23}\right) \tan \theta_{14} \sin \theta_{24}
\end{array}\right), \\
A_{\theta_{13}}^{-}=\sin \theta_{14} \cos \theta_{23} \cos \theta_{24}\left(\begin{array}{cc}
0 & -e^{i\left(\phi_{14}-\phi_{13}\right)} \\
e^{-i\left(\phi_{14}-\phi_{13}\right)} & 2 i \sin \left(\phi_{14}+\phi_{23}-\phi_{13}-\phi_{24}\right) \tan \theta_{23} \sin \theta_{24}
\end{array}\right), \\
A_{\theta_{14}}^{+}=\sin \theta_{24}\left(\begin{array}{cc}
0 & -e^{i\left(\phi_{14}-\phi_{24}\right)} \\
e^{-i\left(\phi_{14}-\phi_{24}\right)} & 0
\end{array}\right), \quad A_{\theta_{14}}^{-}=\left(\begin{array}{ll}
0 & 0 \\
0 & 0
\end{array}\right), \\
A_{\theta_{23}}^{+}=\left(\begin{array}{ll}
0 & 0 \\
0 & 0
\end{array}\right), \quad A_{\theta_{23}}^{-}=\sin \theta_{24}\left(\begin{array}{cc}
0 & -e^{i\left(\phi_{24}-\phi_{23}\right)} \\
e^{-i\left(\phi_{24}-\phi_{23}\right)} & 0
\end{array}\right), \\
A_{\theta_{24}}^{+}=\left(\begin{array}{ll}
0 & 0 \\
0 & 0
\end{array}\right), \quad A_{\theta_{24}}^{-}=\left(\begin{array}{ll}
0 & 0 \\
0 & 0
\end{array}\right) .
\end{gathered}
$$

The matrix elements of $A_{\phi_{13}}$ are given by

$$
\begin{aligned}
& \left(A_{\phi_{13}}^{+}\right)_{11}=-i \sin ^{2} \theta_{13} \cos ^{2} \theta_{14}, \\
& \left(A_{\phi_{13}}^{+}\right)_{12}=-\frac{1}{2} i e^{i\left(\phi_{13}-\phi_{23}\right)} \sin 2 \theta_{13} \sin \theta_{23} \cos \theta_{14} \cos \theta_{24}+\frac{1}{2} i e^{-i\left(\phi_{14}-\phi_{24}\right)} \sin ^{2} \theta_{13} \sin 2 \theta_{14} \sin \theta_{24}, \\
& \left(A_{\phi_{13}}^{+}\right)_{21}=\left(A_{\phi_{13}}^{+}\right)_{12}^{*}, \\
& \left(A_{\phi_{13}}^{+}\right)_{22}=i \sin ^{2} \theta_{13} \sin ^{2} \theta_{23} \cos ^{2} \theta_{24}-i \sin ^{2} \theta_{13} \sin ^{2} \theta_{24} \sin ^{2} \theta_{14} \\
& +\frac{1}{2} i \cos \left(\phi_{23}-\phi_{13}+\phi_{14}-\phi_{24}\right) \sin 2 \theta_{13} \sin \theta_{14} \sin \theta_{23} \sin 2 \theta_{24}, \\
& \left(A_{\phi_{13}}^{-}\right)_{11}=i \sin ^{2} \theta_{13} \cos ^{2} \theta_{23} \\
& \left(A_{\phi_{13}}^{-}\right)_{12}=-\frac{1}{2} i e^{-i\left(\phi_{23}-\phi_{24}\right)} \sin ^{2} \theta_{13} \sin 2 \theta_{23} \sin \theta_{24}+\frac{1}{2} i e^{-i\left(\phi_{13}-\phi_{14}\right)} \sin 2 \theta_{13} \sin \theta_{14} \cos \theta_{23} \cos \theta_{24}, \\
& \left(A_{\phi_{13}}^{-}\right)_{21}=\left(A_{\phi_{13}}^{-}\right)_{12}^{*} \\
& \left(A_{\phi_{13}}^{-}\right)_{22}=i \sin ^{2} \theta_{13} \sin ^{2} \theta_{23} \sin ^{2} \theta_{24}-i \sin ^{2} \theta_{13} \sin ^{2} \theta_{14} \cos ^{2} \theta_{24} \\
& -\frac{1}{2} i \cos \left(-\phi_{14}+\phi_{13}+\phi_{24}-\phi_{23}\right) \sin 2 \theta_{13} \sin \theta_{14} \sin \theta_{23} \sin 2 \theta_{24}, \\
& A_{\phi_{14}}^{+}=i \sin ^{2} \theta_{14}\left(\begin{array}{cc}
-1 & -e^{i\left(\phi_{14}-\phi_{24}\right)} \operatorname{ctan} \theta_{14} \sin \theta_{24} \\
-e^{-i\left(\phi_{14}-\phi_{24}\right)} \operatorname{ctan} \theta_{14} \sin \theta_{24} & \sin ^{2} \theta_{24}
\end{array}\right), \\
& A_{\phi_{14}}^{-}=i \sin ^{2} \theta_{14} \cos ^{2} \theta_{24}\left(\begin{array}{ll}
0 & 0 \\
0 & 1
\end{array}\right) \text {, } \\
& A_{\phi_{23}}^{+}=i \sin ^{2} \theta_{23} \cos ^{2} \theta_{24}\left(\begin{array}{cc}
0 & 0 \\
0 & -1
\end{array}\right), \\
& A_{\phi_{23}}^{-}=i \sin ^{2} \theta_{23}\left(\begin{array}{cc}
1 & e^{i\left(\phi_{24}-\phi_{23}\right)} \operatorname{ctan} \theta_{23} \sin \theta_{24} \\
e^{-i\left(\phi_{24}-\phi_{23}\right)} \operatorname{ctan} \theta_{23} \sin \theta_{24} & -\sin ^{2} \theta_{24}
\end{array}\right), \\
& A_{\phi_{24}}^{+}=i \sin ^{2} \theta_{24}\left(\begin{array}{cc}
0 & 0 \\
0 & -1
\end{array}\right), \quad A_{\phi_{24}}^{-}=i \sin ^{2} \theta_{24}\left(\begin{array}{cc}
0 & 0 \\
0 & 1
\end{array}\right) \text {. }
\end{aligned}
$$




\section{B. Field strength $F$}

In this subsection we shall calculate the field strength $F$ associated with the previous connections $A$. Its components are given by $F_{\mu \nu}=\partial_{\mu} A_{\nu}-\partial_{\nu} A_{\mu}+\left[A_{\mu}, A_{\nu}\right]$. Here, we shall restrict on the field strength components for which the commutator part $\left[A_{\mu}, A_{\nu}\right]$ is zero for all values of the parameters $\theta_{i j}$ and $\phi_{i j}$. For them the computation of their holonomies is straightforward as we shall see in the following subsection. Let us present first the $(\theta, \phi)$ field strength components. The ones that are obtained from commuting connection components acting on the $S_{+}$and $S_{-}$eigenspaces are given by

$$
\begin{aligned}
& \left(F_{\theta_{24} \phi_{13}}^{+}\right)_{11}=0 \\
& \left(F_{\theta_{24} \phi_{13}}^{+}\right)_{12}=\frac{i}{2} e^{i\left(\phi_{13}-\phi_{23}\right)} \sin 2 \theta_{13} \sin \theta_{23} \cos \theta_{14} \sin \theta_{24}+\frac{i}{2} e^{-i\left(\phi_{14}-\phi_{24}\right)} \sin 2 \theta_{14} \sin ^{2} \theta_{13} \cos \theta_{24}, \\
& \left(F_{\theta_{24} \phi_{13}}^{+}\right)_{21}=\left(F_{\theta_{24} \phi_{13}}^{+}\right)_{12}^{*}, \\
& \left(F_{\theta_{24} \phi_{13}}^{+}\right)_{22}=-i \sin \theta_{13} \sin 2 \theta_{24}\left(\sin ^{2} \theta_{14}+\sin ^{2} \theta_{23}\right)+i \cos \left(\phi_{23}-\phi_{13}+\phi_{14}-\phi_{24}\right) \sin 2 \theta_{13} \sin \theta_{14} \sin \theta_{23} \cos 2 \theta_{24}, \\
& \left(F_{\theta_{24} \phi_{13}}^{-}\right)_{11}=0 \\
& \left(F_{\theta_{24} \phi_{13}}^{-}\right)_{12}=-\frac{i}{2} e^{i\left(\phi_{23}-\phi_{24}\right)} \sin ^{2} \theta_{13} \sin 2 \theta_{23} \cos \theta_{24}-\frac{i}{2} e^{i\left(\phi_{13}-\phi_{14}\right)} \sin 2 \theta_{13} \sin \theta_{14} \cos \theta_{23} \sin \theta_{24}, \\
& \left(F_{\theta_{24} \phi_{13}}^{-}\right)_{21}=\left(F_{\theta_{24} \phi_{13}}^{-}\right)_{12}^{*}, \\
& \left(F_{\theta_{24} \phi_{13}}^{-}\right)_{22}=i \sin ^{2} \theta_{13} \sin 2 \theta_{24}\left(\sin ^{2} \theta_{23}+\sin ^{2} \theta_{14}\right)-i \cos \left(\phi_{13}-\phi_{14}+\phi_{24}-\phi_{23}\right) \sin 2 \theta_{13} \sin \theta_{14} \sin \theta_{23} \cos 2 \theta_{24}, \\
& F_{\theta_{24} \phi_{14}}^{+}=\left(\begin{array}{cc}
0 & -\frac{1}{2} i e^{i\left(\phi_{14}-\phi_{24}\right)} \sin 2 \theta_{14} \cos \theta_{24} \\
-\frac{1}{2} i e^{-i\left(\phi_{14}-\phi_{24}\right)} \sin 2 \theta_{14} \cos \theta_{24} & i \sin ^{2} \theta_{14} \sin 2 \theta_{24}
\end{array}\right), \\
& F_{\theta_{24} \phi_{14}}^{-}=-i \sin ^{2} \theta_{14} \sin 2 \theta_{24}\left(\begin{array}{cc}
0 & 0 \\
0 & 1
\end{array}\right), \\
& F_{\theta_{24} \phi_{23}}^{+}=i \sin ^{2} \theta_{23} \sin 2 \theta_{24}\left(\begin{array}{cc}
0 & 0 \\
0 & 1
\end{array}\right) \\
& F_{\theta_{24} \phi_{23}}^{-}=\left(\begin{array}{cc}
0 & \frac{1}{2} i e^{i\left(\phi_{24}-\phi_{23}\right)} \sin 2 \theta_{23} \cos \theta_{24} \\
\frac{1}{2} i e^{i\left(-\phi_{24}+\phi_{23}\right)} \sin 2 \theta_{23} \cos \theta_{24} & -i \sin ^{2} \theta_{23} \sin 2 \theta_{24}
\end{array}\right), \\
& F_{\theta_{24} \phi_{24}}^{+}=-i \sin 2 \theta_{24}\left(\begin{array}{cc}
0 & 0 \\
0 & 1
\end{array}\right), \quad F_{\theta_{24} \phi_{24}}^{-}=i \sin 2 \theta_{24}\left(\begin{array}{cc}
0 & 0 \\
0 & 1
\end{array}\right),
\end{aligned}
$$

From the connection components where only the ones that act on $S_{+}$commute we obtain the following field strength components

$$
\begin{aligned}
F_{\theta_{23} \phi_{13}}^{+}= & \frac{i}{2} \cos \theta_{24} \cos \theta_{23} \sin 2 \theta_{13} \\
& \times\left(\begin{array}{cc}
0 & -e^{i\left(\phi_{13}-\phi_{23}\right)} \cos \theta_{14} \\
-e^{-i\left(\phi_{13}-\phi_{23}\right)} \cos \theta_{14} & 2 \tan \theta_{13} \sin \theta_{23}+2 \cos \left(\phi_{23}-\phi_{13}+\phi_{14}-\phi_{24}\right) \sin \theta_{14} \sin \theta_{24}
\end{array}\right),
\end{aligned}
$$




$$
F_{\theta_{23} \phi_{23}}^{+}=-i \cos ^{2} \theta_{24} \sin 2 \theta_{23}\left(\begin{array}{cc}
0 & 0 \\
0 & 1
\end{array}\right)
$$

while $F_{\theta_{23} \phi_{14}}^{+}=F_{\theta_{23} \phi_{24}}^{+}=0$. From the connection components where only the ones that act on $S_{-}$commute we obtain the following field strength components

$$
\begin{aligned}
F_{\theta_{14} \phi_{13}}^{-}= & -\frac{i}{2} \sin 2 \theta_{13} \cos \theta_{14} \cos \theta_{24} \\
& \times\left(\begin{array}{cc}
0 & -e^{-i\left(\phi_{13}-\phi_{14}\right)} \cos \theta_{23} \\
-e^{i\left(\phi_{13}-\phi_{14}\right)} \cos \theta_{23} & 2 \tan \theta_{13} \sin \theta_{14} \cos \theta_{24}+2 \cos \left(\phi_{13}-\phi_{14}+\phi_{24}-\phi_{23}\right) \sin \theta_{23} \sin \theta_{24}
\end{array}\right), \\
& F_{\theta_{14} \phi_{14}}^{-}=i \cos ^{2} \theta_{24} \sin 2 \theta_{14}\left(\begin{array}{cc}
0 & 0 \\
0 & 1
\end{array}\right),
\end{aligned}
$$

while $F_{\theta_{14} \phi_{23}}^{-}=F_{\theta_{14} \phi_{24}}^{-}=0$. Finally, the $(\theta, \theta)$ field strength components are given by

$$
\begin{aligned}
& F_{\theta_{13} \theta_{24}}^{+}=\sin \theta_{23} \cos \theta_{14}\left(\begin{array}{cc}
0 & -e^{i\left(\phi_{13}-\phi_{23}\right)} \sin \theta_{24} \\
e^{-i\left(\phi_{13}-\phi_{23}\right)} \sin \theta_{24} & -2 i \sin \left(\phi_{13}-\phi_{14}+\phi_{24}-\phi_{23}\right) \tan \theta_{14} \cos 2 \theta_{24}
\end{array}\right), \\
& F_{\theta_{13} \theta_{24}}^{-}=\sin \theta_{14} \cos \theta_{23}\left(\begin{array}{cc}
0 & -e^{i\left(\phi_{14}-\phi_{13}\right)} \sin \theta_{24} \\
e^{-i\left(\phi_{14}-\phi_{13}\right)} \sin \theta_{24} & -2 i \sin \left(\phi_{14}+\phi_{23}-\phi_{13}-\phi_{24}\right) \tan \theta_{23} \cos 2 \theta_{24}
\end{array}\right), \\
& F_{\theta_{14} \theta_{23}}^{+}=F_{\theta_{14} \theta_{23}}^{-}=0 \\
& F_{\theta_{14} \theta_{24}}^{+}=-\cos \theta_{24}\left(\begin{array}{cc}
0 & -e^{i\left(\phi_{14}-\phi_{24}\right)} \\
e^{-i\left(\phi_{14}-\phi_{24}\right)} & 0
\end{array}\right), \quad F_{\theta_{14} \theta_{13}}^{-}=0 \\
& F_{\theta_{23} \theta_{24}}^{+}=0 \quad F_{\theta_{23} \theta_{24}}^{-}=-\cos \theta_{24}\left(\begin{array}{cc}
0 & -e^{i\left(\phi_{24}-\phi_{23}\right)} \\
e^{-i\left(\phi_{24}-\phi_{23}\right)} & 0
\end{array}\right), \\
& F_{\theta_{13} \theta_{23}}^{+}=-\cos \theta_{23} \cos \theta_{14} \cos \theta_{24}\left(\begin{array}{cc}
0 & -e^{i\left(\phi_{13}-\phi_{23}\right)} \\
e^{-i\left(\phi_{13}-\phi_{23}\right)} & 2 i \sin \left(\phi_{13}-\phi_{14}+\phi_{24}-\phi_{23}\right) \tan \theta_{14} \sin \theta_{24}
\end{array}\right), \\
& F_{\theta_{13} \theta_{14}}^{-}=-\cos \theta_{14} \cos \theta_{23} \cos \theta_{24}\left(\begin{array}{cc}
0 & -e^{i\left(\phi_{14}-\phi_{13}\right)} \\
e^{-i\left(\phi_{14}-\phi_{13}\right)} & 2 i \sin \left(\phi_{14}+\phi_{23}-\phi_{13}-\phi_{24}\right) \tan \theta_{23} \sin \theta_{24}
\end{array}\right) .
\end{aligned}
$$

\section{Holonomies $\Gamma$}

In general, the explicit calculation of the Holonomies of matrix connections $A$ is non-straightforward as they involve the path ordering procedure when exponentiating their loop integral. On the other hand it is possible to restrict our cyclic evolutions to specific planes $\left(\sigma, \sigma^{\prime}\right)$ that correspond to commuting components $A_{\sigma}$ and $A_{\sigma^{\prime}}$. For those loops we can employ Stokes theorem for the abelian theories and obtain

$$
\Gamma_{A}^{ \pm}(C) \equiv \mathbf{P} \exp \oint_{C} A^{ \pm}=\exp \oint_{C} A^{ \pm}=\exp \iint_{\Sigma(C)} d \sigma d \sigma^{\prime} F_{\sigma \sigma^{\prime}}^{ \pm}\left(\sigma, \sigma^{\prime}\right)
$$

where the rest of the variables are considered constant. The path ordering symbol has been taken out at the second step as the connection components on the $\left(\sigma, \sigma^{\prime}\right)$ commute with each other. Hence, Stokes theorem can be applied straightforwardly as presented in the previous section for the abelian Berry phase. An analytic expression for the holonomies can be obtained by exponentiating the $2 \times 2$ matrices resulting from the surface integral of the field strength components presented in the previous subsection. 


\section{DISCUSSION}

A theoretical model has been presented for the construction of non-abelian holonomies for the $G(4,2)$ Grassmannian manifold. This is the generalization of the usual abelian Berry phase to the case of a quantum system consisting of a doubly degenerate energy eigenspace. The evolution of both degenerate spaces has been presented that are produced from the same cyclic adiabatic evolution. The main difference with the abelian case is that it is possible to have manipulations of state population in each degenerate space rather than just generation of overall phase factors. This can be achieved for example by spanning loops $C$ on the $\left(\theta_{24}, \phi_{13}\right)$ plane where population can be interchanged in a well defined way between the states $|1\rangle$ and $|2\rangle$ as well as between $|3\rangle$ and $|4\rangle$. In contrast, loops on the $\left(\theta_{24}, \phi_{24}\right)$ plane contribute only Berry-like phases on the conjugate states $|2\rangle$ and $|4\rangle$ (see Figure 1). It is worth noticing that the holonomic evolution is not producing any correlations between the two different eigenspaces $S_{+}$and $S_{-}$due to the cyclicity of the adiabatic procedure. Even though it is possible to have each degenerate eigenspace evolving with a different holonomy there is a correspondence between the evolutions as can be easily seen in the previous section facilitating eventually their detection in a physical system and the verification of the above results. Indeed, the components $F^{+}$and $F^{-}$of the field strength have a similar functional dependence on the variables $\left(\theta_{i j}, \phi_{i j}\right)$ and their surface integral in (5) bears a common dependence in the area $\Sigma(C)$. These holonomic characteristics can be verified in the laboratory with present technology by employing an atomic cloud and manipulating the atomic states with external laser beams.

[1] M. V. Berry, Proc. R. Soc. London Ser. A 392, 45 (1984).

[2] F. Wilczek and A. Zee, Phys. Rev. Lett. 52, 2111 (1984); for a review see, Geometric Phases in Physics, A. Shapere and F. Wilczek, Eds. World Scientific, 1989.

[3] P. Zanardi, and M. Rasetti, Phys. Lett. A 264, 94 (1999); S. Seshadri, S. Lakshmibala, and V. Balakrishnan, Phys. Rev. A 55, 869 (1997); K. Fujii, "Introduction to Grassmann Manifolds and Quantum Computation", to appear in J. of Appl. Math., quant-ph/0103011; E. Ercolessi, G. Marmo, G. Morandi, N. Mukunda, "Geometry of mixed states and degeneracy structure of geometric phases for multi-level quantum systems. A unitary group approach", quant-ph/0105007.

[4] M. Nakahara, Geometry, Topology and Physics, IOP Publishing Ltd. (1990).

[5] J. Pachos, P. Zanardi, and M. Rasetti, Phys. Rev. A $61010305(R)$, (2000).

[6] J. Pachos, and S. Chountasis, Phys. Rev. A 62, 052318 (2000).

[7] L. M. Duan, J. I. Cirac, and P. Zoller, Science 292, 1695 (2001).

[8] J. Pachos, and H. Walther, "Quantum computation with trapped ions in an optical cavity", Phys. Rev. Lett. 89 187903-1 (2002); A. Recati, T. Calarco, P. Zanardi, J. I. Cirac, and P. Zoller, "Holonomic quantum computation with neutral atoms", quant-ph/0204030.

[9] P. Solinas, P. Zanardi, N. Zanghi, and F. Rossi, "Non-adiabatic geometrical quantum gates in semiconductor quantum dots", quant-ph/0301089; "Holonomic quantum gates: A semiconductor-based implementation", quant-ph/0301090.

[10] M. S. Gulley, A. G. White, and D. F. V. James, "A Raman approach to quantum logic in Calcium-like ions", quant$\mathrm{ph} / 0112117$ and references therein.

[11] For a generalization of the abelian Stokes theorem to the non-abelian case see: R. L. Karp, F. Mansouri, and J. S. Rno, J. Math. Phys. 406033 (1999).

[12] I. Fuentes-Guridi, J. Pachos, S. Bose, V. Vedral, S. Choi, Phys. Rev. A 66, 022102 (2002).

[13] R. G. Unanyan, B. W. Shore, and K. Bergmann, Phys. Rev. A 59, 2910 (1999).

[14] J. Pachos and P. Zanardi, Int. J. Mod. Phys. B 15, 1257 (2001).

[15] J. Pachos, "Quantum Computation by Geometrical Means", published in the AMS Contemporary Math Series volume entitled "Quantum Computation and Quantum Information Science", quant-ph/0003150. 